\title{
Are Mechanically Ventilated Patients with COVID-19 More Likely to Die Than Those without COVID-19? Perhaps Not
}

\author{
Jigeeshu V Divatia
}

Keywords: Acute hypoxemic respiratory failure, APACHE IV, COVID-19 mortality, Mechanical ventilation, Mortality, Pneumonia. Indian Journal of Critical Care Medicine (2021): 10.5005/jp-journals-10071-24065

Since December 2019, the world has been in the throes of COVID-19, the disease caused by the SARS-CoV-2 virus, with more than 250 million cases and over 5 million deaths. India has reported over 34 million cases and 4,60,791 deaths. ${ }^{1}$ About $5 \%$ of all patients and $20 \%$ of hospitalized patients with COVID-19 may experience severe manifestations, such as pneumonia with acute hypoxemic respiratory failure (AHRF) or acute respiratory distress syndrome (ARDS), sepsis, and multi-organ failure necessitating intensive care unit (ICU) admission. ${ }^{2}$ Mortality of COVID-19 patients admitted to the ICU is high. In one meta-analysis, $31 \%$ of patients admitted to the ICU died, ${ }^{3}$ while in another, mortality ranged from 0 to $84.6 \%$, with a pooled mortality of $35.5 \%$, and a trend of reducing mortality over time. $^{4}$

The need for mechanical ventilation has been associated with high mortality. In a meta-analysis of 28 studies comprising 12,437 COVID-19ICU admissions from seven countries, mortality in patients receiving invasive mechanical ventilation (IMV) was 43\%, and ICU and IMV duration were 7.78 and 10.12 days, respectively. ${ }^{5}$ In the RECOVERY trial of dexamethasone vs standard treatment, mortality in mechanically ventilated patients was $41 \%$ in the usual care arm and $29 \%$ in patients receiving dexamethasone. ${ }^{6}$ In the RECOVERY trial comparing tocilizumab vs usual care, 28-day mortality was 49 vs $51 \%$ for ventilated patients receiving tocilizumab or usual care, respectively. ${ }^{7}$ In a large European cohort study that included 4,244 adult patients with severe COVID-19 admitted to the ICU, mortality 90 days after ICU admission was 37\% in the subgroup of patients who received invasive mechanical ventilation on the day of ICU admission. ${ }^{8}$ Among these patients, mortality increased with the severity of ARDS at ICU admission (30, 34, and 50\% for mild, moderate, and severe ARDS, respectively). Thus, mortality in patients with COVID-19 receiving IMV appears to be approximately 30-50\%.

The key question is whether patients with COVID-19 receiving IMV are more likely to die than other patients requiring IMV for AHRF? The answer to this question is not of mere academic interest; excessive mortality could lead to denial of IMV to some patients in the setting of a pandemic and an upsurge in the cases of COVID-19 or early end-of-life care decisions. Clinicians would feel reluctant to offer IMV and offer a hopeless prognosis if a patient with COVID-19 went on to require IMV even in the absence of a major surge in cases.

Comparisons with mortality in similar patients prior to the pandemic would result in erroneous conclusions. During the pandemic, there were considerable disruptions of hospital services. Hospital and ICU beds dedicated to the treatment of COVID-19 patients were created to provide for surge capacity. This resulted in a shortage of trained staff and resources to manage critically ill
Department of Anaesthesia, Critical Care and Pain, Tata Memorial Hospital, Homi Bhabha National Institute, Mumbai, Maharashtra, India Corresponding Author: Jigeeshu V Divatia, Department of Anaesthesia, Critical Care and Pain, Tata Memorial Hospital, Homi Bhabha National Institute, Mumbai, Maharashtra, India, Phone: +91 9869077435, e-mail: jdivatia@yahoo.com

How to cite this article: Divatia JV. Are Mechanically Ventilated Patients with COVID-19 More Likely to Die Than Those without COVID-19? Perhaps Not. Indian J Crit Care Med 2021;25(12):1341-1342.

Source of support: Nil

Conflict of interest: None

patients, especially in makeshift ICUs and in existing ICUs where capacity was ramped up. Thus, comparisons with historical controls, either from the same hospital or the same country, or comparisons with the global literature would not provide reliable information.

The most reliable comparison would be that of mortality in COVID-19 and non-COVID-19 patients receiving IMV for AHRF treated concurrently over the same period. Thus, the study by Ghosh and Todi in this issue of the IJCCM is important. ${ }^{9}$ They prospectively studied a cohort of 48 COVID-19 and 95 non-COVID-19 ICU patients who were mechanically ventilated for AHRF between March 28, 2020, and November 30, 2020 (during India's "first wave"). The study methodology is notable on several counts. All patients on mechanical ventilation during the study period were analyzed till they were extubated or had expired, thus avoiding incomplete data due to patients being categorized as "still in hospital." Further, they used the Acute Physiology and Chronic Health Evaluation (APACHE) IV scoring system, a standard ICU severity of illness scoring system to risk-stratify patients, obtain a risk-adjusted mortality rate, and estimate the standardized mortality ratio (SMR). ${ }^{10}$ Use of the APACHE IV system thus facilitates comparisons of the severity of illness between the COVID-19 and non-COVID-19 patients. The WHO severity of illness scoring system ${ }^{11}$ used in many studies is probably too crude and not granular enough to assess the severity of disease in patients already admitted to the ICU and receiving IMV. Ghosh and Todi found that crude ICU and hospital mortality were similar in COVID-19 patients and their non-COVID-19 counterparts ( 43.8 vs $40 \%$ and 43 vs $41.1 \%$, respectively). There were no significant differences in terms of risk-adjusted ICU and hospital mortality between the two groups. Mechanically ventilated COVID-19 patients had longer ICU stay and more days on ventilation compared to mechanically ventilated non-COVID-19 patients admitted during the same period. ${ }^{10}$ The mortality in COVID-19

(c) The Author(s). 2021 Open Access This article is distributed under the terms of the Creative Commons Attribution 4.0 International License (https://creativecommons. org/licenses/by-nc/4.0/), which permits unrestricted use, distribution, and non-commercial reproduction in any medium, provided you give appropriate credit to the original author(s) and the source, provide a link to the Creative Commons license, and indicate if changes were made. The Creative Commons Public Domain Dedication waiver (http://creativecommons.org/publicdomain/zero/1.0/) applies to the data made available in this article, unless otherwise stated. 
patients and the non-COVID-19 patients is comparable with that in the world literature.

The single-center design, while making the study less generalizable, overcomes many confounding factors while testing the hypothesis. The capacity to provide adequate numbers of doctors, nurses, technicians, and paramedical staff capable of managing critically ill patients requiring IMV was severely strained and aggravated by travel restrictions due to the nationwide lockdown, staff shortages due to the healthcare providers being infected by the virus, and quarantine of high-risk contacts to varying degrees in different hospitals and different parts of the country. Expertise, equipment, ICU admission policies, and protocols for instituting and managing IMV varied in different parts of the country and from hospital to hospital even within the same city. Analyzing a cohort from a single center where more uniform patient management practices can be assumed overcomes some of this confounding.

The authors compared the SMR using predicted mortality in the COVID-19 cohort with that in the entire cohort of non-COVID-19 patients with AHRF. Perhaps a comparison with 22 patients with non-COVID-19 viral pneumonia may have provided greater insight. In a large American multicenter study, Higgins et al. compared 1,491 patients with COVID-19 and 4,200 patients with a diagnosis of non-coronavirus disease viral pneumonia receiving ICU care. ${ }^{12}$ Contrary to Ghosh and Todi, they found that COVID-19 was associated with higher risk-adjusted mortality (hospital SMR was 1.52 for COVID-19 patients and 0.82 for viral pneumonia patients).

Further, staff shortages, lack of trained staff, and the pressures of working during surges of cases in a pandemic can affect the care of both COVID-19 as well as non-COVID-19 patients. While the observed mortality in all patients is well within the global range of mortality, it would be interesting to see whether the risk-adjusted mortality in the authors' ICU was similar or worse than that before the pandemic. The data from this study may not apply to centers without comparable expertise, equipment, and organization of services as in the authors' hospital and during massive surges of patients as occurred during the second wave in India. Also, all the data reported in the study pertain to the first wave and most likely exclude disease caused by the SARS-CoV-2 delta variant which is currently the predominant variant in India.

With the above caveats, this study serves to generate the hypothesis that mortality in COVID-19 patients may not be different from that in non-COVID-19 patients with AHRF. The data from this study should give confidence to clinicians working in ICU setups similar to that of authors that mortality in COVID-19 patients is not prohibitively high, and that the outcome and prognosis for COVID-19 patients requiring IMV is similar to that of non-COVID-19 patients who receive IMV. Hence, systematic avoidance of IMV in COVID-19 patients is not warranted, and COVID-19 patients who develop severe AHRF must receive IMV when clinically indicated.

\section{OrCID}

Jigeeshu V Divatia @ https://orcid.org/0000-0001-7384-4886

\section{REFERENCES}

1. Available from: https://www.worldometers.info/coronavirus/ [Last accessed on November 7, 2021].

2. Wiersinga WJ, Rhodes A, Cheng AC, Peacock SJ, Prescott HC. Pathophysiology, transmission, diagnosis, and treatment of coronavirus disease 2019 (COVID-19): a review. JAMA 2020;324(8):782793. DOI: 10.1001/jama.2020.12839.

3. Abate SM, Ali SA, Mantfardo B, Basu B. Rate of intensive care unit admission and outcomes among patients with coronavirus: a systematic review and meta-analysis. PLoS One 2020;15(7):e0235653. DOI: 10.1371/journal.pone.0235653.

4. Armstrong RA, Kane AD, Cook TM. Outcomes from intensive care in patients with COVID-19: a systematic review and meta-analysis of observational studies. Anaesthesia 2020;75(10):1340-1349. DOI: 10.1111/anae.15201.

5. Chang R, Elhusseiny KM, Yeh Y-C, Sun WZ. COVID-19 ICU and mechanical ventilation patient characteristics and outcomes - a systematic review and meta-analysis. PLoS One 2021;16(2):e0246318. DOI: $10.1371 /$ journal.pone.0246318.

6. RECOVERY Collaborative Group, Horby P, Lim WS, Emberson JR, Mafham M, Bell JL, et al. Dexamethasone in hospitalized patients with Covid-19. N Engl J Med 2021;384(8):693-704. DOI: 10.1056/ NEJMoa2021436.

7. RECOVERY Collaborative Group. Tocilizumab in patients admitted to hospital with COVID-19 (RECOVERY): a randomised, controlled, open-label, platform trial. Lancet 2021;397(10285):1637-1645. DOI: 10.1016/S0140-6736(21)00676-0.

8. COVID-ICU Group on behalf of the REVA Network and the COVID-ICU Investigators. Clinical characteristics and day-90 outcomes of 4244 critically ill adults with COVID-19: a prospective cohort study. Intensive Care Med 2021;47(1):60-73. DOI: 10.1007/s001 34-020-06294-x.

9. Todi S, Ghosh S. A Comparative Study on the Outcomes of Mechanically Ventilated COVID-19 vs Non-COVID-19 Patients with Acute Hypoxemic Respiratory Failure. Indian J Crit Care Med 2021;25(12):1377-1381.

10. Zimmerman JE, Kramer AA, McNair DS, Malila FM. Acute Physiology and Chronic Health Evaluation (APACHE) IV: hospital mortality assessment for today's critically ill patients. Crit Care Med 2006;34(5):1297-1310. DOI: 10.1097/01.CCM.0000215112.84523.F0.

11. Screening, triage and clinical assessment: early recognition of patients with COVID-19. World Health Organisation update 1.4 clinical management of COVID-19: living guidance. Available from: https:// app.magicapp.org/\#/guideline/j1WBYn [Last accessed November 7, 2021].

12. Higgins TL, Stark MM, Henson KN, Freeseman-Freeman L. Coronavirus disease 2019 ICU patients have higher-than-expected acute physiology and chronic health evaluation-adjusted mortality and length of stay than viral pneumonia ICU patients. Crit Care Med 2021;49(7):e701-e706. DOI: 10.1097/CCM.0000000000005012. 